\section{Acetylphosphatase in Animal Tissues}

IN the course of experiments on the metabolism of acetylphosphate in rats, an enzyme which catalyses the hydrolysis of this substrate was found. The enzyme is present in homogenates as well as in cellfree extracts of liver, muscle, kidney, brain, but not in blood serum. Acetylphosphate $M / 20$ (prepared according to Lipmann and Tuttle ${ }^{1}$ ) was incubated with liver homogenate and sodium bicarbonate buffer $(p \mathrm{H} 7 \cdot 3)$ at $38^{\circ} \mathrm{C}$. More than 80 per cent cleavage was observed in five minutes, and total cleavage was obtained in fifteen minutes. The spontaneous cleavage of acetylphosphate in the same conditions but in the absence of liver was 33 per cent in 180 minutes. The enzyme activity was found to be of the same order of magnitude in extracts of liver, kidney, brain and muscle.

Some properties of the enzyme are described below. The experiments were conducted at $25^{\circ} \mathrm{C}$. in order to slow up the action. The hydrolysis was followed (a) by estimation of the amounts of inorganic phosphate liberated, using the method of Lipmann and Tuttle ${ }^{2},(b)$ volumetrically in terms of carbon dioxide formed in the presence of sodium bicarbonate, due to the liberation of acid equivalents during cleavage.

The activity of the extracts was completely destroyed by boiling for five minutes. Acetylphosphatase is relatively resistant to fluoride poisoning. No inhibition was observed with concentrations of $M / 100$ sodium fluoride. $M / 20$ sodium fluoride retarded the hydrolysis by 50 per cent (as measured by the amount of substrate hydrolysed after fifteen minutes). Sodium cyanide failed altogether to influence the enzyme activity even at concentrations of $M / 10$. On the other hand, inorganic phosphate is strongly inhibiting. Inorganic phosphate appears to compete with acetylphosphate for the enzyme : thus, at a substrate concentration of $M / 100$, a concentration of $M / 30$ inorganic phosphate caused complete inhibition, whereas at $M / 20$ substrate concentration an inhibition of only 60 per cent was obtained. Enzyme activity at this substrate concentration is completely annulled by $M / 10$ inorganic phosphate, the hydrolysis proceeding at the same rate as in the absence of enzyme.

The non-identity of acetylphosphatase with ordinary 'alkaline' phosphatase is shown by the different distribution of these enzymes in organs and their different behaviour with poisons. The presence of a highly active acetylphosphatase in animal tissues suggests an explanation for negative results obtained with the use of this substrate for phosphate transfer ${ }^{3}$ and acetylation ${ }^{4}$ in animal tissues. It seems probable that when acetylphosphate is added to animal tissues it is removed by hydrolysis before its possible action as a phosphate or acetyl donor can become manifest. When acetylphosphate appears as an intermediate in metabolism, it is probably maintained in an adequate steady-state concentration within the tissue because the action of acetylphosphatase in these conditions is retarded by inorganic phosphate.

\section{B. Shaprro. \\ E. WeRTHEIMER.}

Laboratory for Pathological Physiology,

Hebrew University, Jerusalem.

${ }^{1}$ Lipmann, F., and Tuttle, L. C., J. Biol. Chem., 153, 577 (1944).

'Lipmann, F., and Tuttle, L. C., J. Biol. Chem., 153, 571 (1944).

Ochoa, S., Peters, R. A., and Stocken, L. A., Nature, 144, 750 (1939).

- Lipmann, F., Fed. Proc., 4, 97 (1945).

\section{An Attempt to Obtain Nuclear Excitation by Means of X-Rays}

WhEN a substance is irradiated with $\gamma$-rays of an energy less than that necessary for disintegration of the nuclei (for most of the elements about $8 \mathrm{MeV}$.), only a nuclear excitation can take place. If radioactivity is obtained by such an irradiation, it is necessary to assume the existence of isomeric metastable states to which the nuclei have been excited and from which transitions to the groundlevel take place by emission of (highly converted) $\gamma$-rays. Experiments in this direction were performed in 1939 by Pontecorvo ${ }^{1}$ and Collins ${ }^{2}$. They succeeded in activating indium by means of $\mathrm{X}$-rays with an energy of more than $1 \mathrm{MeV}$. By direct irradiation of eleven elements, Trumpy ${ }^{3}$ later claimed to have ascertained the existence of thirty-five new isomeric nuclei with characteristic half-life periods. By varying the tube voltage from 300 to $1,800 \mathrm{kV}$., Trumpy was able to determine the critical excitation energy with an accuracy of 10 per cent. The values were surprisingly low and in fourteen cases less than $0 \cdot 4 \mathrm{MeV}$.

With an X-ray tube supplied from a high-tension equipment for a $400 \mathrm{kV}$. maximum, I have irradiated aluminium, copper, rhodium, silver, cadmium, indium, tin, gold and lead. As the radiation emitted by the transition to the ground-level must be assumed to be rather weak, the counters are made of the element which is under investigation. In some cases, however, the substances (in the form of thick foils) have been investigated inside a specially constructed counter where the radiation from the active substance is only absorbed by the gas in the counter chamber.

The X-ray tube has worked at $390 \mathrm{kV}$. (max.) with a tube current of $5 \mathrm{~mA}$. (max.). The anti-cathode is of tungsten and in the shape of a disk, so that about half the current is reflected backwards.

On the irradiation of copper, rhodium, cadmium, indium, tin, gold and lead, activity has in no case been observed. With a Geiger-Müller tube of aluminium made four years ago, a weak effect of the same order of magnitude as the zero effect was observed, the activity decreasing with a half-life period of about 2-3 min. Freshly made tubes or foils of aluminium do not show this effect. A Geiger-Müller tube of glass with a cathode of silver also showed a weak activity of the same intensity as the aluminium tube.

The negative result with rhodium is especially interesting as the nucleus ${ }^{103} \mathrm{Rh}$ is excited by irradiation with fast neutrons ${ }^{4}$ to an isomeric state of the stable nucleus and situated only $19 \mathrm{kV}$. above the ground-level. For such an element, according to our present conception of the formation of isomeric nuclei by high-voltage $X$-rays, the activation levels might be expected to be situated nearer the groundlevel than is the case when the lowest metastable state lies, for example, $200 \mathrm{kV}$. above the groundlevel.

At the present stage, when the voltage of the tube cannot be increased, it can only be said that, with the intensity and energy of the $\mathrm{X}$-ray quanta used here, no activation levels can be found in the elements nam $\epsilon \mathrm{d}$ above. As pointed out by Wiedenbeck ${ }^{5}$ and Northrup et al. ${ }^{6}$ spocial care must be taken in investigations with irradiated Geiger-Müller tubes. Sherrer ${ }^{7}$ has shown that in special circumstances field emission of electrons can take place from the Giger-Müller tube cathode surface, thus falsifying an induced activity. It seems probable to me that 